Revista Monografias Ambientais - REMOA v. 15, n.1, jan-abr. 2016, p.264-271

Revista do Centro de Ciências Naturais e Exatas - UFSM, Santa Maria

\title{
ANÁLISE DA PRODUÇÃO CIENTÍFICA DOS ÚLTIMOS ANOS SOBRE MUDANÇAS CLIMÁTICAS E MEIO AMBIENTE
}

\author{
Analysis of The Scientific Production in the Last Years of the Climate Change and Environment \\ Juliana Benitti Lorenzett ${ }^{1}$ e Daniel Benitti Lorenzett ${ }^{2}$ \\ ${ }^{1}$ Curso de Engenharia Ambiental, UFSM, Santa Maria, RS, Brasil \\ 2 Engenharia de Produção, UFSM, Santa Maria, RS, Brasil
}

\section{Resumo}

O objetivo deste artigo situa-se na análise da produção científica sobre mudanças climáticas publicada nos últimos 06 anos, verificando as abordagens e metodologias de pesquisa utilizadas na área. Para desenvolvimento da pesquisa, utilizaramse as abordagens qualitativa e quantitativa combinadas. A busca de dados foi realizada por meio do portal de periódicos CAPES, utilizando como parâmetro para busca os seguintes termos: "Mudanças climáticas" e "Meio Ambiente". Os resultados apontam que a produção científica nacional dos últimos anos pode ser considerada metodologicamente incipiente, pois os artigos são empíricos qualitativos, realizados sem rigor metodológico para validação dos dados analisados.

Palavras-chave: Mudanças climáticas. produção do conhecimento. produção científica.

Abstract

The aim of this paper lies in the analysis of scientific literature on climate change published over the last 06 years, checking approaches and research methodologies in the area. To develop the research, we used the combined qualitative and quantitative approaches. The search for data was performed through the portal of CAPES, using as a parameter to search the following terms: "Climate change" and "Environment". The results show that the national scientific production in recent years can be considered methodologically incipient because the articles are qualitative empirical, conducted without rigorous methodology for validation of the data analyzed.

Keywords: Climate change. Production of knowledge. Scientific production. 


\section{Introdução}

Avaliar o volume de publicações de uma determinada área, instituição ou pesquisador consiste em analisar a produção científica. A análise da produção do conhecimento científico nas mais diversas áreas, se tornou tarefa freqüente em muitos estudos, pois ela permite o entendimento de como determinada área de conhecimento vem se desenvolvendo ao longo dos anos. Por meio desse acompanhamento, é possível realizar uma avaliação dos resultados e questionar os diferentes aspectos da produção científica (ALVES, 2009; MARCELO e HAYASHI, 2013).

Essas Análises permitem conhecer o padrão de crescimento e distribuição das publicações entre os periódicos, para ordenar e visualizar seus aspectos metodológicos e conceituais a partir dos quais o tema está sendo discutido, além de contribuírem para traçar um importante panorama da abordagem científica, evidenciando tendências e lacunas na produção científica nacional, sinalizando caminhos para pesquisas futuras (AMBIEL e POLLI, 2011; ANDRIGHI et al., 2011; MANDÚ ET. AL, 2012).

O presente estudo se propõe a analisar a produção do conhecimento sobre mudanças climáticas e meio ambiente dos últimos 06 anos, pois estudos e contribuições originais na área podem trazer novas perspectivas e novos conhecimentos.

Mediante a necessidade de verificar a tendência metodológica da produção acadêmica brasileira sobre a temática "mudanças climáticas", surge a seguinte questão de pesquisa: Quais são as características da produção científica brasileira dos últimos 06 anos, na área de mudanças climáticas e meio ambiente?

Nesse sentido, torna-se necessário, especificamente, levantar os periódicos nacionais qualificados no WebQualis que publicaram artigos sobre mudanças climáticas nos últimos 06 anos, analisando quais são os pesquisadores e instituições de pesquisa da área e quais são suas abordagens e metodologias de pesquisa.

A presente pesquisa servirá para demonstrar o enfoque dos trabalhos, e a metodologia utilizada pelos pesquisadores nacionais que publicaram sobre o assunto, sinalizando tendências para trazer novas perspectivas e novos campos de estudo.

\section{Metodologia da pesquisa}

Esta pesquisa no que se refere à abordagem, foi classificada como qualitativa, pois os dados obtidos na pesquisa não receberam tratamento estatístico, foram interpretados de forma subjetiva, buscando esclarecer a percepção e entendimento sobre a natureza geral do tema em estudo, abrindo espaço para a interpretação dos autores (BRYMAN, 1989; GIL, 2009; CAUCHICK MIGUEL et al., 2010).

Para o desenvolvimento dessa pesquisa adotou-se, como critério, analisar artigos científicos nacionais publicados nos últimos 06 anos em periódicos relacionados no portal de periódicos da CAPES. Como critério de busca no portal de periódicos CAPES, foram utilizados os termos "Mudanças Climáticas" e "Meio Ambiente", usando o conector "AND", selecionando para análise somente artigos nacionais publicados em língua portuguesa, entre os anos de 2010 e 2015.

A busca pelos artigos, na plataforma de periódicos CAPES, ocorreu em outubro de 2015. Foram encontrados 12 artigos (FIGURA 01), como resultado da busca. Entretanto, 05 artigos foram excluídos da amostra, pois um dos artigos era na realidade um dossiê e os outros 04 artigos eram internacionais. Restaram, na amostra final, 07 artigos a serem analisados. 


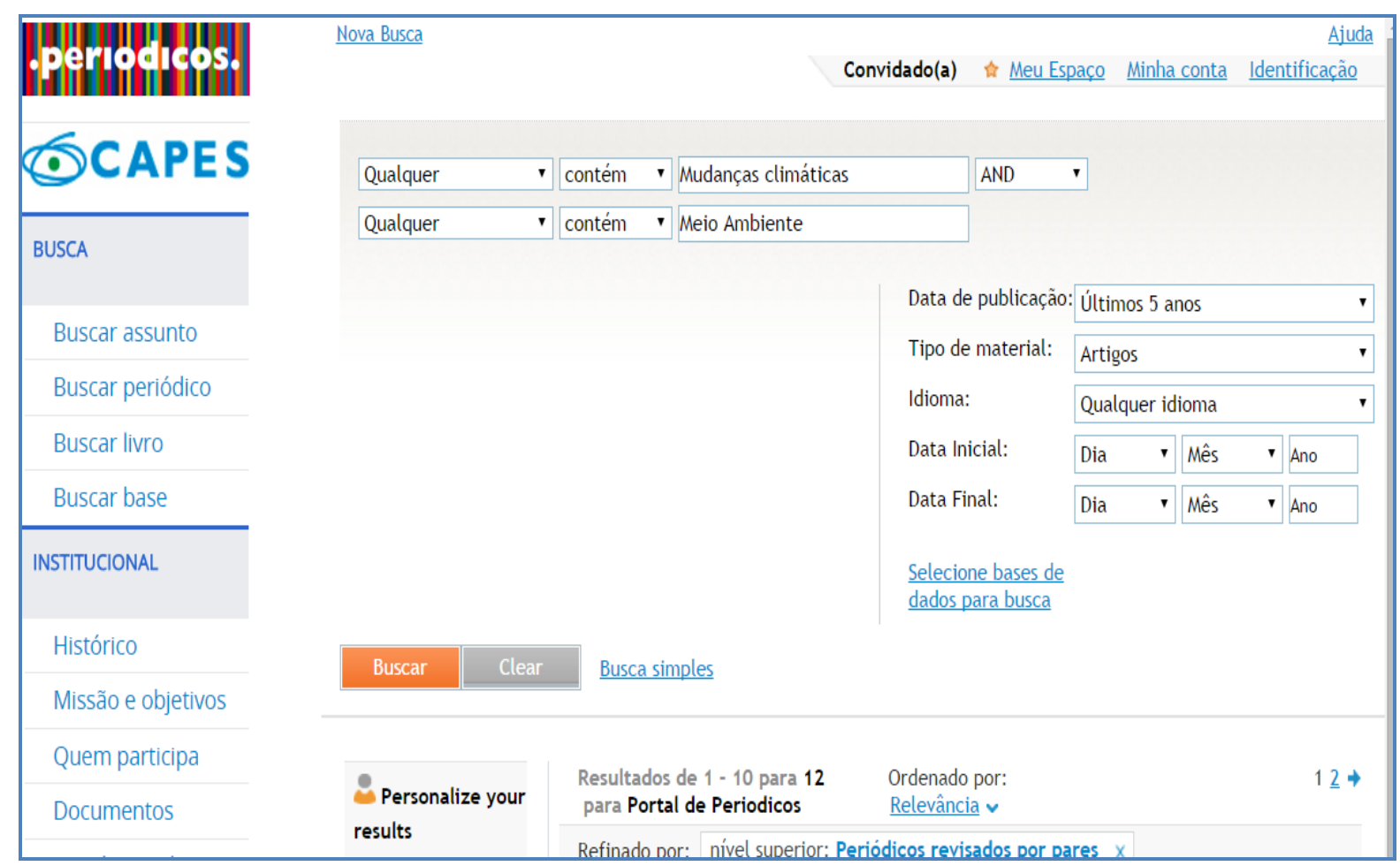

Figura 01 - Pesquisa no portal de periódicos CAPES

Para análise dos artigos da amostra, procedeu-se a leitura de seus títulos, resumos, palavraschaves, introdução e metodologia. Os dados obtidos foram tabulados com o auxílio do Microsoft Excel®, e analisados por meio de gráficos.

\section{Análise e discussão dos dados}

Iniciando a análise, foi elaborado o Quadro 1, onde consta uma descrição dos dados levantados, contendo o nome do periódico, a instituição a qual o periódico está vinculado, a classificação no WebQualis CAPES 2014 para Engenharias I, o total de artigos publicados nesse período, e o total de artigos encontrados que continham os termos de busca.

\begin{tabular}{|l|l|c|c|c|c|}
\hline Nome do periódico & Instituição & $\begin{array}{c}\text { Qualis CAPES } \\
\text {-Eng. I (2014) }\end{array}$ & $\begin{array}{c}\text { Total de artigos a } \\
\text { partir de 2010 }\end{array}$ & $\begin{array}{c}\text { Artigos } \\
\text { Encontrados }\end{array}$ & \% \\
\hline Rev. Geográfica Acadêmica & UFRR & B4 & 74 & 1 & $1,35 \%$ \\
\hline REGE & USP & NC* & 180 & 1 & $0,56 \%$ \\
\hline Ambiente \& Sociedade & USP & B2 & 179 & 1 & $0,56 \%$ \\
\hline Ciên. \& Saúde Coletiva & ABRASCO & NC* & 2075 & 3 & $0,14 \%$ \\
\hline RA'E GA & UFPR & B5 & 206 & 1 & $0,49 \%$ \\
\hline Total & & $\mathbf{2 7 1 4}$ & $\mathbf{7}$ & $\mathbf{0 , 2 6 \%}$ \\
\hline
\end{tabular}

*NC - Não classificada para Engenharias I

Quadro 1 - Periódicos que publicaram artigos enquadrados nos critérios da pesquisa Fonte: Pesquisa (2015)

Conforme pode ser observado no Quadro 1, foram identificados 05 periódicos, acessíveis por meio eletrônico que publicaram artigos sobre mudanças climáticas no período em análise. Juntos, esses periódicos publicaram um total de 2.714 artigos. Deste total, somente 07 artigos continham os termos de busca da pesquisa, ou seja, $0,26 \%$ do total de artigos publicados. 
O periódico "Ciência \& Saúde Coletiva" apresenta o maior número de artigos publicados sobre a temática, no entanto, fazendo a relação entre o volume de publicações das revistas e o número de artigos sobre mudanças climáticas que elas publicaram, observa-se nitidamente que o periódico "Rev. Geográfica Acadêmica" se destaca, apresentando o maior volume de publicações sobre a temática, cerca de 1,35\% do seu volume total de trabalhos publicados.

Observa-se, também, que todos os periódicos analisados são qualificados pela CAPES. Entretanto, as revistas REGE e Ciências \& Saúde Coletiva não são classificados para a área de Engenharias I. Sendo os demais periódicos apresentados da categoria B. Dessa forma, é possível afirmar que os artigos analisados estão concentrados em revistas de baixo impacto.

Os artigos pesquisados são de autoria de 14 autores diferentes, e nenhum desses autores possuía mais de uma publicação entre os artigos analisados, não sendo possível estabelecer uma "hierarquia" de autoria na área.

$\mathrm{Na}$ análise referente ao tipo de publicação (individual ou em grupo), verificou-se a predominância de publicações com formação de grupos de pesquisa. Conforme se observa no Gráfico 1, merece destaque as publicações em duplas, que representam $43 \%$ das publicações no período entre os anos de 2010 e 2015.

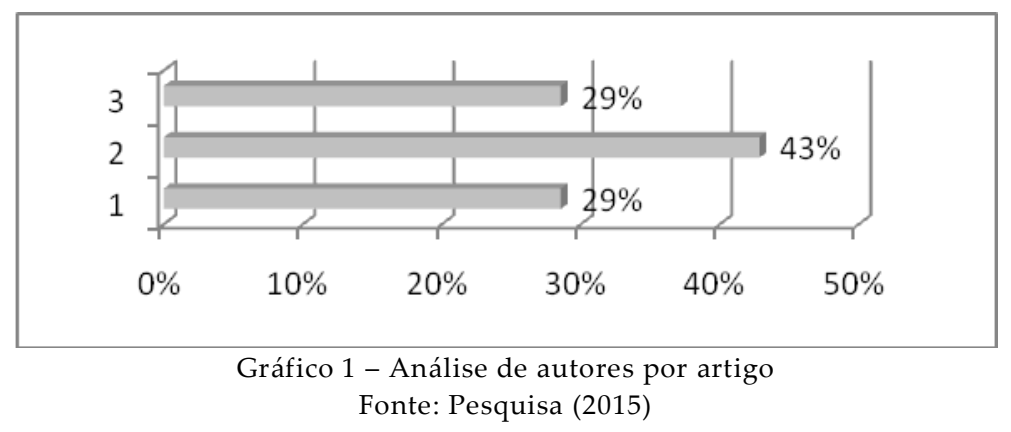

Observa-se, conforme Gráfico 2, com relação ao anos de publicação, que o maior volume de publicação está centralizado no ano de 2012, que apresentou 4 publicações na área. Tendo havido uma lacuna de publicações na área nos anos de 2013 e 2014, nos quais não houve nenhuma publicação.

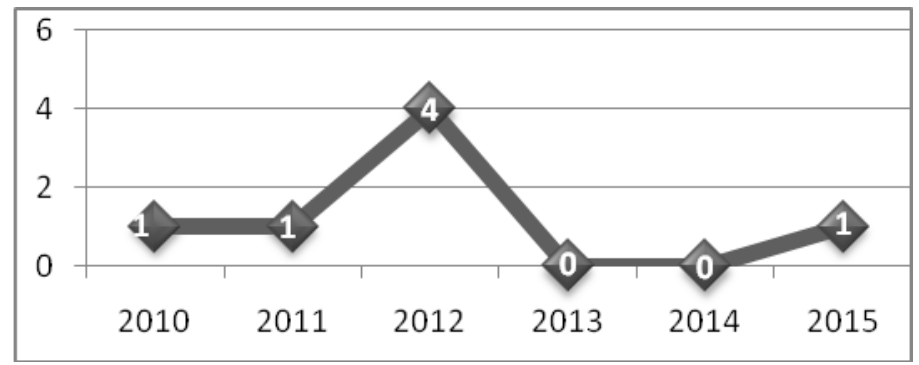

Gráfico 2 - Tendência das publicações ao longo dos anos Fonte: Pesquisa (2015)

A primeira parte da análise dos artigos processou-se por meio da tabulação dos artigos que resultou no Quadro 2, onde consta o ano de publicação, nome do periódico, instituição a qual o periódico pertence, título do artigo, e respectivos autores. Uma vez tabulados procedeu-se a leitura dos títulos, palavras-chave e resumos dos artigos. 


\begin{tabular}{|c|c|c|c|c|c|}
\hline Ano & Art & Nome do periódico & Título do artigo & Instituição & Autores \\
\hline$\stackrel{ }{\overline{1}}$ & 1 & $\begin{array}{l}\text { Rev. Geográfica } \\
\text { Acadêmica }\end{array}$ & $\begin{array}{l}\text { Governanca climatica nas cidades: reduzindo } \\
\text { vulnerabilidades e aumentando resiliencia. }\end{array}$ & UNICAMP & Rafael D'Almeida Martins \\
\hline$\overline{\bar{I}}$ & 2 & REGE & $\begin{array}{l}\text { Evidenciação das operações com crédito de carbono nos } \\
\text { relatórios da administração e nas notas explicativas. }\end{array}$ & FEA-USP & $\begin{array}{l}\text { Vanderlei dos Santos; Ilse Maria Beuren; } \\
\text { Rita Buzzi Rausch }\end{array}$ \\
\hline \multirow{4}{*}{ 곡 } & 3 & Ambiente \& Sociedade & $\begin{array}{l}\text { Análise da vulnerabilidade da microrregião de Itajubá por } \\
\text { meio do IVG com vistas à mitigação dos impactos } \\
\text { causados pelas mudanças climáticas. }\end{array}$ & UNIFEI & $\begin{array}{l}\text { Luiz Henrique Tibúrcio; Marcelo de Paula } \\
\text { Corrêa }\end{array}$ \\
\hline & 4 & Ciên. \& Saúde Coletiva & $\begin{array}{l}\text { Resíduos sólidos urbanos: impactos socioambientais e } \\
\text { perspectiva de manejo sustentável com inclusão social. }\end{array}$ & USP & Nelson Gouveia \\
\hline & 5 & Ciên. \& Saúde Coletiva & $\begin{array}{l}\text { Abordagens ecossistêmica e comunicativa na implantação } \\
\text { de agendas territorializadas de desenvolvimento } \\
\text { sustentável e promoção da saúde. }\end{array}$ & FIOCRUZ & $\begin{array}{l}\text { Edmundo Gallo; Andréia Faraoni Freitas } \\
\text { Setti }\end{array}$ \\
\hline & 6 & RA'E GA & $\begin{array}{l}\text { Considerações sobre a desconstrução do licenciamento } \\
\text { ambiental brasileiro. }\end{array}$ & USP & $\begin{array}{l}\text { Alexandre Cosme José JERONYMO; Célio } \\
\text { BERMANN; Sinclair Mallet Guy GUERRA }\end{array}$ \\
\hline$\stackrel{\text { กิ }}{\frac{1}{1}}$ & 7 & Ciên. \& Saúde Coletiva & $\begin{array}{l}\text { Índices sintéticos de vulnerabilidade: uma revisão } \\
\text { integrativa de literatura. }\end{array}$ & UnB & $\begin{array}{l}\text { Lívia Rejane Miguel Amaral Schumann; } \\
\text { Leides Baroso Azevedo Moura }\end{array}$ \\
\hline
\end{tabular}

Quadro 2 - Artigos que se enquadraram nos critérios da pesquisa

Fonte: Pesquisa (2015)

Analisando o Quadro 2, observa-se que a principal instituição de pesquisa na área é a USP, pois publicou 02 artigos sobre tema analisado.

Os 07 artigos analisados apresentaram um total de 31 palavras-chave, mas não foi possível traçar uma tendência das publicações na área por meio dessa metodologia, pois não houve ocorrência de repetição de nenhuma das palavras-chave nos artigos analisados.

Analisando as referências dos artigos, verificou-se que os 07 artigos estudados apresentaram um total de 279 referências. Observa-se pelo Gráfico 3, que a tendência ao longo dos anos das publicações foi pela adoção de referências nacionais, pois elas representaram $63 \%$ do total das referências encontradas nos artigos.

Com relação à tendência das referências, no que se refere ao veículo de publicação. As referências dos artigos provêm de livros, artigos publicados em revistas e eventos, de teses e dissertações, e também de outros veículos de comunicação, como relatórios técnicos e legislação. Nessa análise ganha destaque a utilização de referencias de revistas, que representou $41 \%$ das fontes utilizadas pelos autores da área. Merece destaque, ainda, a utilização de outras fontes, com $32 \%$ do total das referências. Isso de deve ao fato da maioria dos artigos ter utilizado a metodologia de pesquisa documental, para a coleta de dados.

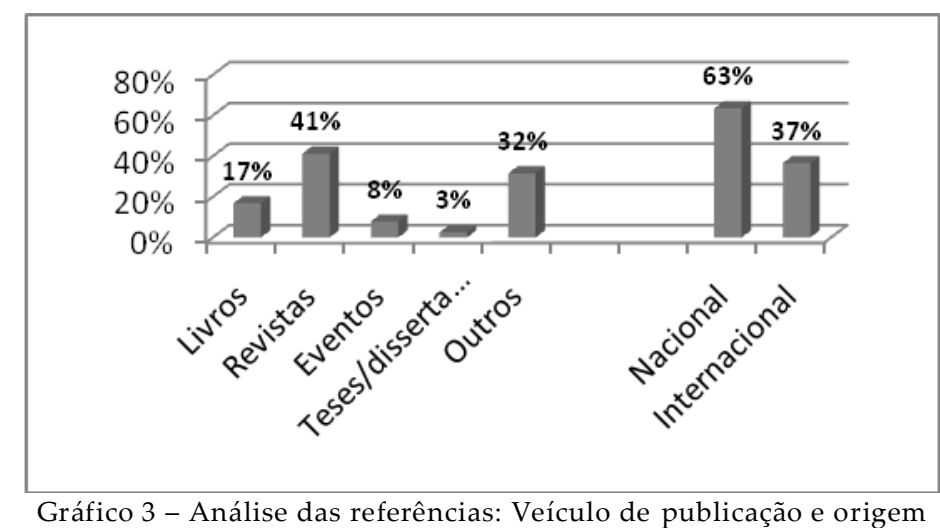

Fonte: Pesquisa (2015)

Não foi possível estabelecer uma tendência quanto ao uso das fontes ao longo dos anos, como se observa no Gráfico 4, o uso de fontes de revistas se destacou nos anos de 2010 e 2015, e o uso de outras fontes bibliográficas se destacou nos anos de 2011 e 2012. Já com relação à nacionalidade das referências, elas iniciam em uma tendência inversamente proporcional e a 
partir de 2011 as referências nacionais passam a se manter a cima das internacionais de forma constante.

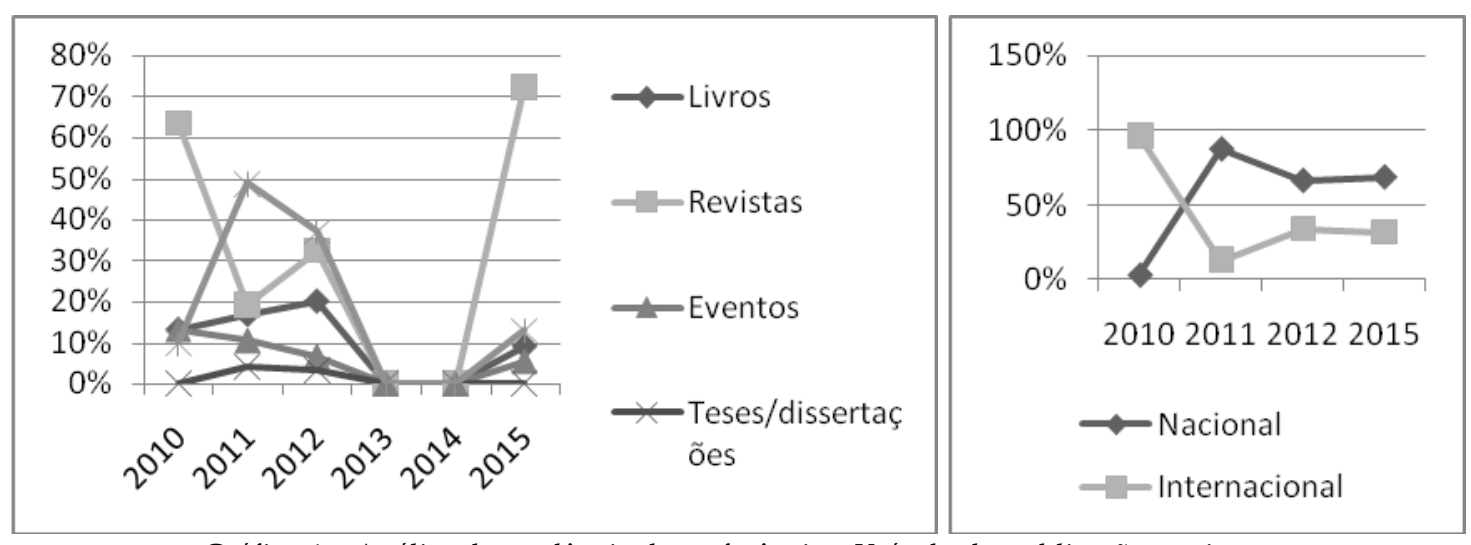

Gráfico 4 - Análise da tendência das referências: Veículo de publicação e origem Fonte: Pesquisa (2015)

Para análise de conteúdo dos artigos foi elaborado o Quadro 3, onde são apresentados os estudos realizados em cada um dos 07 artigos da amostra. Os estudos sobre mudanças climáticas estão delimitados nas seguintes áreas: Análise da produção científica; mercado de crédito de carbono; vulnerabilidade socioambiental; resíduos sólidos, saúde; e projetos do setor hidrelétrico. Merecendo destaque os trabalhos de revisão de literatura na área de análise da produção científica sobre mudanças climáticas.

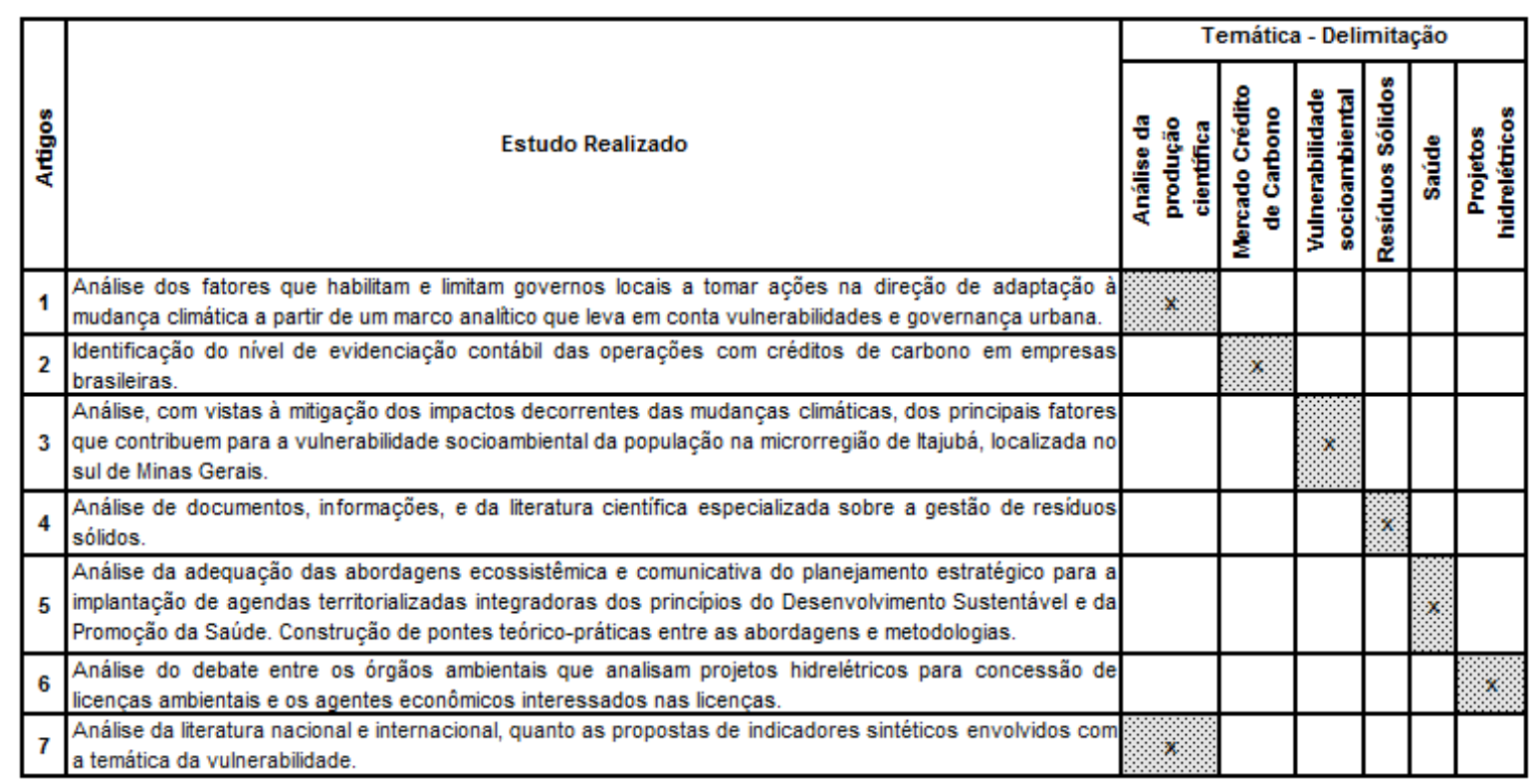

Quadro 3 - Estudos realizados por segmento Fonte: Pesquisa (2015)

Na leitura da seção de metodologia dos artigos, verificou-se a tendência pela elaboração de estudos com enfoque empírico e abordagem qualitativa. Dos 07 artigos analisados somente um apresentou abordagem quantitativa, conforme se observa no Quadro 4.

A maioria dos estudos realizados utilizou a pesquisa bibliográfica e a documental para a coleta de dados, sem rigor metodológico para validação dos dados apresentados. 


\begin{tabular}{|c|c|c|c|c|c|c|c|c|c|c|c|c|c|c|c|c|c|c|c|}
\hline \multirow[b]{2}{*}{ 总 } & \multicolumn{2}{|c|}{ Estudo } & \multicolumn{7}{|c|}{ Método } & \multicolumn{4}{|c|}{ Coleta de dados } & \multicolumn{2}{|c|}{ Objetivo } & \multicolumn{2}{|c|}{\begin{tabular}{|l|} 
Justificativa \\
\end{tabular}} & \multicolumn{2}{|c|}{ Problema } \\
\hline & $\underset{\text { 总 }}{\stackrel{\Xi}{\Xi}}$ & 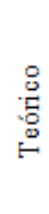 & 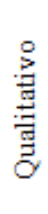 & 胥 & 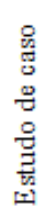 & 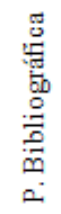 & 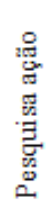 & 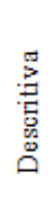 & 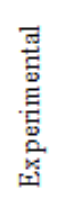 & 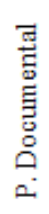 & 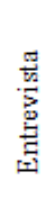 & 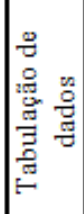 & 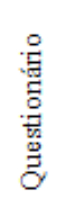 & 具 & z & 㰴 & z & 具 & z \\
\hline 1 & - & & - & & & - & & & & & & m & & m & & m & & 1 & \\
\hline 2 & 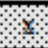 & & - & & & & & 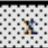 & & 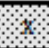 & & & & 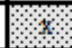 & & 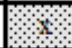 & &  & \\
\hline 3 & - & & & 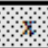 & मे & & & & & - & & & & - & & m & & m & \\
\hline 4 & 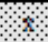 & & 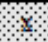 & & & - & & & & 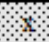 & & & & - & & x & & 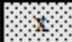 & \\
\hline 5 & - & & - & & & r & 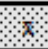 & & & - & & & & ra & & m & & 2 & \\
\hline 6 & 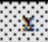 & & - & & & 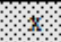 & & & & + & & & & 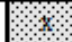 & & x & & . & \\
\hline 7 & - & & - & & & - & & & & & & $\alpha$ & & - & & a & & n & \\
\hline
\end{tabular}

Quadro 4 - Estudos realizados por Análise da estrutura e metodologia utilizadas Fonte: Pesquisa (2015)

Por meio da leitura da introdução dos artigos, identificou-se a existência de problema, objetivo e justificativa alinhados em todos os artigos analisados. Entretanto, na maioria dos artigos esses elementos não estavam estruturados, eram implícitos ou subjetivos.

\section{Considerações Finais}

Neste estudo analisou-se a produção do conhecimento sobre mudanças climáticas dos últimos 06 anos, verificando que a tendência metodológica da produção acadêmica brasileira na área baseia-se na realização de estudos empíricos de pesquisa bibliográfica e documental, com abordagem qualitativa. A produção acadêmica não é estruturada, pois a maioria dos artigos analisados apresenta objetivo, justificativa e problema de pesquisa de forma implícita e subjetiva.

A tendência para publicação na área teve seu ápice no ano de 2012, não sendo possível estabelecer uma tendência quanto às palavras-chave utilizadas nem quanto aos autores que publicam na área, pois não houve recorrência nas publicações. A produção científica demonstrou-se fundamentada, principalmente, em referências de artigos acadêmicos nacionais e em documentos primários.

Observa-se, desse modo, que a produção científica analisada pode ser considerada metodologicamente incipiente, pois os artigos são empíricos qualitativos, realizados sem rigor metodológico para validação dos dados.

\section{Referências}

ALVES, B. H. Abordagens métricas: análise da produção científica de artigos e rede de colaboração científica dos docentes do programa de pós-graduação em ciência da informação, na linha de pesquisa organização da informação da Unesp/Marília. Revista de Iniciação Científica da FFC. V. 9, № 2, 2009. Disponível em: <http://www2.marilia.unesp.br/revistas/index.php/ric/article/view/248/208>. Acesso em: 13 de jan. 2016.

AMBIEL, Rodolfo Augusto Matteo; POLLI, Mariana Fralleti. Análise da produção científica brasileira sobre avaliação psicológica em orientação profissional. Estudos Interdisciplinares em
Psicologia,
$\mathrm{v}$.
2 ,
n. 1 ,
2011.
Disponível
em:

$<$ http://www.uel.br/revistas/uel/index.php/eip/article/view/10649/9338>. Acesso em: 20 de jan. 2016. 
ANDRIGHI, F.F.; HOFFMANN, V.E.; ANDRADE, M.A.R. Análise da produção científica no campo de estudo das redes em periódicos nacionais e internacionais. Revista de Administração e Inovação, v. 8, n. 1, p. 29-54, 2011. Disponível em: <http://www.revistarai.org/rai/article/view/530/pdf_33>. Acesso em: 13 de setembro de 2015.

BRYMAN, A. Research methods and organization studies.London: UnwinHyman, 1989.

CAUCHICK MIGUEL, P. A. (Coord.), FleURY, Afonso, MELlO, C. E. P., NAKANO, D. N., TURRIONI, J. B., HO, L. L., MORABITO, R., MARTINS, R. A., \& PUREZA, V. Metodologia de pesquisa em engenharia de produção e gestão de operações. Rio de Janeiro: Elsevier, 2010.

GIL, A. C. Como elaborar projetos de pesquisa. 4. ed., 12. Reimp. São Paulo: Atlas, 2009.

HABBERMAS, Jurgen. Mudança estrutural da esfera pública: investigações quanto a uma categoria da sociedade burguesa. Trad. Flá- vio R. Kotche. 2. ed. Rio de Janeiro: Tempo Brasileiro, 2003.

MANDÚ, Edir Nei Teixeira; PEDUZZI, Marina; SILVA, Ana Maria Nunes. Análise da produção científica nacional sobre o trabalho de enfermagem. Revista Enfermagem UERJ, V. 20, № 1, 2012. Disponível em: <http://www.epublicacoes.uerj.br/index.php/enfermagemuerj/article/view/4005/2774>. Acesso em: 15 de jan. 2016.

MARCELO, J.F.; HAYASHI, M.C.P.I. Estudo bibliométrico sobre a produção científica no campo da sociologia da ciência. Informação \& Informação, v. 18, n. 3, p. 138-153, 2013. Disponível em: $<$ http://www.uel.br/revistas/uel/index.php/informacao/article/view/8413/pdf_2>. Acesso em: 15 de outubro de 2015. 